\title{
DISCUSIÓN DE LA APLICABILIDAD DEL DESARROLLO SOSTENIBLE DESDE LAS POLÍTICAS PÚBLICAS EN LA REPÚBLICA DOMINICANA Y EN COLOMBIA SEGÚN LA ADAPTACIÓN EDUCATIVA DE LA AGENDA 2030
}

\section{Discussion of the applicability of sustainable development from public policies in the Dominican Republic and Colombia according to the educational adaptation of the 2030 Agenda}

\section{Jacqueline Murillo Garnica}

Instituto Superior de Formación Docente Salomé Ureña, República Dominicana

Correo-e: jacqueline.murillo@isfodosu.edu.do ORCID: 0000-0003-31625131

Recibido: 02/5/2019 • Aprobado: 20/5/2019

Cómo citar: Murillo Garnica, J. (2019). Discusión de la aplicabilidad del desarrollo sostenible desde las políticas públicas en la República Dominicana y en Colombia según la adaptación educativa de la agenda 2030. Ciencia y Sociedad, 44(2), 37-51. Doi: https://doi.org/10.22206/cys.2019.v44i2.pp37-51

\section{Resumen}

El desarrollo sostenible con énfasis educativo se ha convertido en un elemento transversal en la agenda global, logrando vincular organizaciones sociales y estatales que se preocupan por el estado actual de los procesos de aprendizaje y de calidad de cada país, buscando continuamente que la adopción, mejoramiento y equilibrio entre la sociedad, los fundamentos económicos, el medio ambiente y la educación ocurran en el menor tiempo posible, en aras de mejorar las condiciones de vida y las relaciones humanas con el entorno. Este trabajo de investigación describe la aplicabilidad del "Desarrollo Sostenible" desde la perspectiva de las políticas públicas en materia de educación para los casos de Colombia y República Dominicana, con relación a los siguientes ejes: acceso, la calidad, la voluntad política y legalidad que tienen ambos países, entendiendo y abordando los enfoques que se le han dado en cada uno de los casos y que permiten que se gestionen reformas políticas, el desarrollo de proyectos internacionales y agendas de concertación multilaterales. Concibiendo las posturas temáticas autónomas de los casos de estudio. El estudio toma datos de fuentes oficiales con tal de mostrar los avances y los retrocesos concernientes a los ejes anteriormente planteados, entendiendo sus particularidades políticas, económicas y sociales, con fuerte incidencia en el desarrollo y adaptación del desarrollo sostenible.

Palabras clave: desarrollo sostenible; Colombia; políticas públicas; República Dominicana; educación.

\begin{abstract}
Sustainable development with educational emphasis has become a cross-cutting element in the global agenda, linking social and state organizations that are concerned about the current state of learning processes and quality of each country, continuously seeking that the adoption, improvement and balance between society, economic fundamentals, the environment and education occur in the shortest time possible, in order to improve living conditions and human relations with the environment. This research work will deal with the applicability of "Sustainable Development" from the perspective of public policies on education for the cases of Colombia and the Dominican Republic, in relation to access, quality, political will and legality that both countries have, understanding and addressing the approaches that have been given in each of the cases and that allow to manage policy reforms, development of international projects and multilateral concertation agendas, conceiving the autonomous thematic positions of the case studies. For this purpose, data will be taken from official sources, which will account for the advances or setbacks in the aforementioned axes, understanding their political, economic and social particularities, with a strong impact on the development and adaptation of Sustainable Development.
\end{abstract}

Keywords: Sustainable Development; Colombia; Public Policies; Dominican Republic; Education. 


\section{Introducción}

El Desarrollo Sostenible (DS) cuenta con un crecimiento histórico fundamentado, principalmente, en organismos internacionales. Para los casos de Colombia y República Dominicana las entidades del orden nacional, territorial y local son las que se encargan de poner en práctica y materializar los lineamientos y directrices que se desarrollan desde el orden internacional; a su vez, el papel de los presidentes ha tenido incidencia sobre el desarrollo y postura adoptada. Sin embargo, diferentes fenómenos internos han logrado frenar el avance de los postulados del DS, como lo son la adaptación a la globalización, el consumo masivo, la concentración de la riqueza, la pobreza multidimensional y la inequidad, entre otros.

Al reconocer las particularidades de estos dos países, se pretenden evidenciar los enfoques y avances que se han desarrollado, tanto desde la visión pública-política, como desde la adaptación de los lineamientos internacionales. Además, los avances multidimensionales sobre las diferentes necesidades y demandas sociales que se aprecian desde la postura educativa resultan ser un tema central para ambos casos, siendo ejes centrales para la construcción y el mejoramiento de las condiciones de vida, respondiendo a los intereses extranjeros y a las necesidades locales.

Aunque, para el caso colombiano como dominicano, lo que se propone es desarrollar una sinergia multisectorial, que vincule activamente a las universidades y centros educativos, para que desde el poder ejecutivo se impulsen iniciativas que se relacionen con la propuesta de la Organización de las Naciones Unidas (ONU) y responda eficaz y eficientemente a las necesidades sociales, frente a la agenda del año 2030 liderada por la Comisión Económica para América Latina y el Caribe (CEPAL) y La Organización de las Naciones Unidas para la Educación, la Ciencia y la Cultura (UNESCO), como ha venido ocurriendo en los casos que pretendemos abordar, los cuales han evidenciado voluntad política, necesidades de mejora y adopción general de las agendas internacionales, siendo posible apreciar la creciente preocupación por parte de estos dos países en la materia.

\section{Orígenes del desarrollo sostenible}

El concepto sobre el DS está fuertemente relacionado a la constante preocupación de la mayoría de los países sobre las relaciones del entorno con los seres humanos, por ello, desde la comunidad internacional, se ha planteado un debate que se consolidó en acuerdos multilaterales hacia las décadas finales del siglo $\mathrm{xx}$ y en acciones concretas en lo que lleva el siglo xxI. Esta situación pretende evidenciar las relaciones existentes entre el desarrollo económico y el desarrollo social sobre los recursos naturales, reconociendo las repercusiones tripartitas en estos ejes fundamentales de la sociedad. Sin embargo, décadas más adelante la nueva discusión se concentra en las consecuencias futuras y condiciones habitacionales de todas las especies sobre el planeta, de ahí que las características propias de esta visión permiten que su vigencia sea renovada constantemente, gracias a la multiplicidad de los actores que influyen en estos temas, tanto a nivel internacional como local.

Como se ha mencionado anteriormente, este proceso de consolidación se dio desde la multidisciplinariedad de enfoques, debido a que las diferentes comisiones de desarrollo permitieron que premios nobel y académicos con diferentes especificidades y formaciones se sumaran al proceso de evaluación y análisis de la situación. El enriquecimiento de esta perspectiva permite cimentar los elementos actuales de lo que hoy se cataloga como DS, condensando las características y elementos esenciales en los informes oficiales de la ONU, para que por medio de su amplia divulgación y reconocimiento se hagan masivos y vinculen a las naciones, incitando a la adopción de lineamientos y recomendaciones que se sugieren. 
La conceptualización sobre el DS no es nueva. Los orígenes de su planteamiento se remontan a la década de los años cincuenta, pero el debate se endurece hacia los años setenta en la medida en que la transformación del entorno se hace más evidente. Las múltiples causas de pobreza aumentan y el fenómeno de las drogas se posiciona y gana espacio en la agenda mundial. Sistemáticamente, durante este periodo, también se adelantan reuniones en donde se abordan temas como el peligro inminente que sufre la biodiversidad, el aumento significativo de la contaminación, la inestabilidad en los programas de seguridad alimentaria, el abuso de los recursos energéticos, entre otros.

Todos los ejes referidos se materializaron en el Informe Brundtland de 1987 denominado como "Nuestro Futuro Común". En este documento se formaliza por primera vez el concepto de DS y se plantea como "el desarrollo que satisface las necesidades de la generación presente sin comprometer la capacidad de las generaciones futuras para satisfacer sus propias necesidades" (Brundtland, 1987, p. 59).

El informe proyecta que el crecimiento económico es y será imperante frente a los recursos naturales y la calidad de vida de los seres humanos. No obstante, si se corrige esta situación se alcanza a vislumbrar la visión positiva y de transformación frente a la reducción de la pobreza, la participación efectiva de las comunidades en el cambio y la conservación social y ambiental por encima de los intereses económicos.

Es decir, que de acuerdo con el Informe Brundtland el DS se fundamenta en tres grandes ejes que articula y funcionan idealmente dentro de cada Estado: la economía, la sociedad y lo medioambiental. Por ello:

El desarrollo sostenible no pone a debate la cuestión de capitalismo o socialismo; del libre mercado o estatismo, sino que a partir del medio ambiente, propone una revolución planificada, pacífica y gradual, que modifique el actual enfoque económico, cultural y social de nuestra relación con la naturaleza y en las relaciones entre la misma sociedad (Treviño \& Sánchez, s. f, párr. 2).

En la actualidad, es posible ver y analizar bajo cuáles preceptos se han incrementado esfuerzos en materia internacional, para lograr hacer sostenibles los ejes sin afectar su desarrollo al interior de las sociedades. La unión entre los gobiernos por medio de o de diferentes estamentos dependientes de la ONU y algunas organizaciones sin ánimo de lucro son los escenarios reales para la transformación y adaptación del desarrollo sostenible al interior de cada país.

Desde la perspectiva de la Organización de las Naciones Unidas para la Educación, la Ciencia y la Cultura (UNESCO), los objetivos vigentes y en los cuales se debe trabajar fuertemente para asegurarlos en el DS son:

- Asegurar un nivel sostenible de población.

- Revivir el crecimiento económico.

- Conservar y reforzar la base de los recursos naturales.

- Unir los aspectos económicos y ambientales en la toma de decisiones.

- Reorientar la tecnología y el manejo de riesgos.

- Conservar y reforzar los recursos naturales.

- Satisfacer las necesidades básicas (agua, energía, sanidad, trabajo).

Finalmente, desde inicios del siglo xxI el DS se ha visto acogido especialmente por los jóvenes, ya que el dinamismo y el nuevo entendimiento de este grupo poblacional se presta para que su aplicación se dé de la mejor manera y en el menor tiempo posible. De este modo, "El reto de la sostenibilidad hay que afrontarlo desde el marco global y el marco local, al 
igual que desde el marco individual" (Antequera, González \& Ríos Osorio, 2005, p. 24).

\section{El desarrollo sostenible en Colombia}

El DS es un tema que se presenta en la agenda política del país. El gobierno ha suscrito varios acuerdos y tratados internacionales que demuestran la voluntad hacia la consecución de objetivos y hacia el mejoramiento de las condiciones en general de los habitantes, pese a que tengan diferentes prioridades e intereses. Esta postura se ha venido implementando lentamente y con apoyo de fundaciones, organizaciones o gobiernos extranjeros que ven en Colombia un foco de oportunidad y cambio en las próximas décadas.

Sin embargo, aún faltan muchos esfuerzos por parte de las entidades y organismos que componen el Gobierno, ya que son ellos quienes canalizan la realidad que vive el país y con este conocimiento buscan proponer proyectos y leyes que permitan solucionar o mitigar los daños que se han generado hasta el día de hoy. En esta sección se podrá ver el DS desde las tres perspectivas que ya hemos planteado anteriormente, para de esta forma analizar su funcionamiento, sinergia, compatibilidad y prospectiva.

\section{Perspectiva política, visión para el 2030}

Desde la perspectiva política podemos decir que Colombia se ha visto involucrada en varios cambios; el principal y más visible en la materia se dio en el año 2011, cuando el recién elegido presidente por aquel entonces, Juan Manuel Santos, divide el Ministerio de Ambiente, Vivienda y Desarrollo Territorial, creando dos entidades encargadas cada una de sus respectivas áreas: el Ministerio de Vivienda y el Ministerio de Medio Ambiente y Desarrollo Sostenible.

En el Plan Nacional de Desarrollo 2014-2018 se contemplan las tres dimensiones del desarrollo, como vía de respuesta a las necesidades de la sociedad colombiana. Parten del bienestar económico, la prosperidad social y la riqueza ambiental. Dicho plan establece posturas de prevención, mejoramiento y construcción del bienestar y beneficio social, como fin último. De este modo, se logra comprometer a las empresas privadas, de hidrocarburos y transporte, para que a través de planes de responsabilidad se mitiguen los riesgos y daños ocasionados por el sector. Estas acciones transversales se apoyan en lineamientos nacionales y se realizan por medio de modelos de rendición de cuentas.

Colombia, en los últimos años, se ha comprometido con el mundo para poder materializar los Objetivos del Desarrollo Sostenible (ODS) planteados por la UNESCO. Estos diecisiete objetivos cuentan con una visión especial en cada caso. Desde las Alcaldías y Gobernaciones se deben diseñar alianzas para el mejoramiento sistemático de las comunidades afectadas. Es importante señalar que tanto los pactos como los proyectos que vinculen el DS son prioritarios y se ejecutarán de manera preferencial con la intención de que los resultados se vean en un menor tiempo.

Además, la visión que se tiene para el año 2030 se enfoca en tres objetivos fundamentales:

Objetivo 1. Avanzar contundentemente hacia un crecimiento sostenible y bajo en carbono.

Objetivo 2. Proteger y asegurar el uso sostenible del capital natural y mejorar la calidad y gobernanza ambiental.

Objetivo 3. Lograr un crecimiento resiliente y reducir la vulnerabilidad frente a los riesgos de desastres y al cambio climático.

En contraste con esta situación el Gobierno Nacional ha tratado de desarrollar planes de acción y asociación entre las demás entidades que podrían implementar el DS desde las políticas públicas y los proyectos vinculados a las colectividades. Un 
ejemplo de ello son los ministerios de Colombia, en especial el Ministerio del Trabajo, el Ministerio de Educación Nacional, el Ministerio de Salud y Seguridad Social y el Ministerio de Medio Ambiente y Desarrollo Sostenible; pero, pese a todos los esfuerzos, se hace muy difícil desarrollar puntos de concertación en las diferentes carteras, ya que cada una cuenta con sus prioridades y especificidad en los sectores económicos que manejan, haciendo que el DS se vea difuso por parte del Gobierno, forzando a trabajar en cada organización minuciosamente y demorando el proceso de construcción y consolidación en Colombia de una visión unificada pública sobre el DS.
En ese orden de ideas, en el último período de tiempo, el crecimiento económico, la estabilidad financiera y la proactividad de los sectores vinculados han dado como resultado que "Colombia sea catalogado como un país de renta media" (CEPAL, 2012 , p. 8), lo cual permite que se le reconozca está desarrollando instituciones sólidas y mejorando la calidad de vida. Por otro lado, Colombia ha buscado la manera de acceder a la Organización para la Cooperación y el Desarrollo Económicos (OCDE), fortalecer la Alianza del Pacífico y adelantar acuerdos de libre comercio, dejando de lado las necesidades sociales y ambientales que enfrenta el país.

\section{Gráfico 1. Ingresos de la economía colombiana}

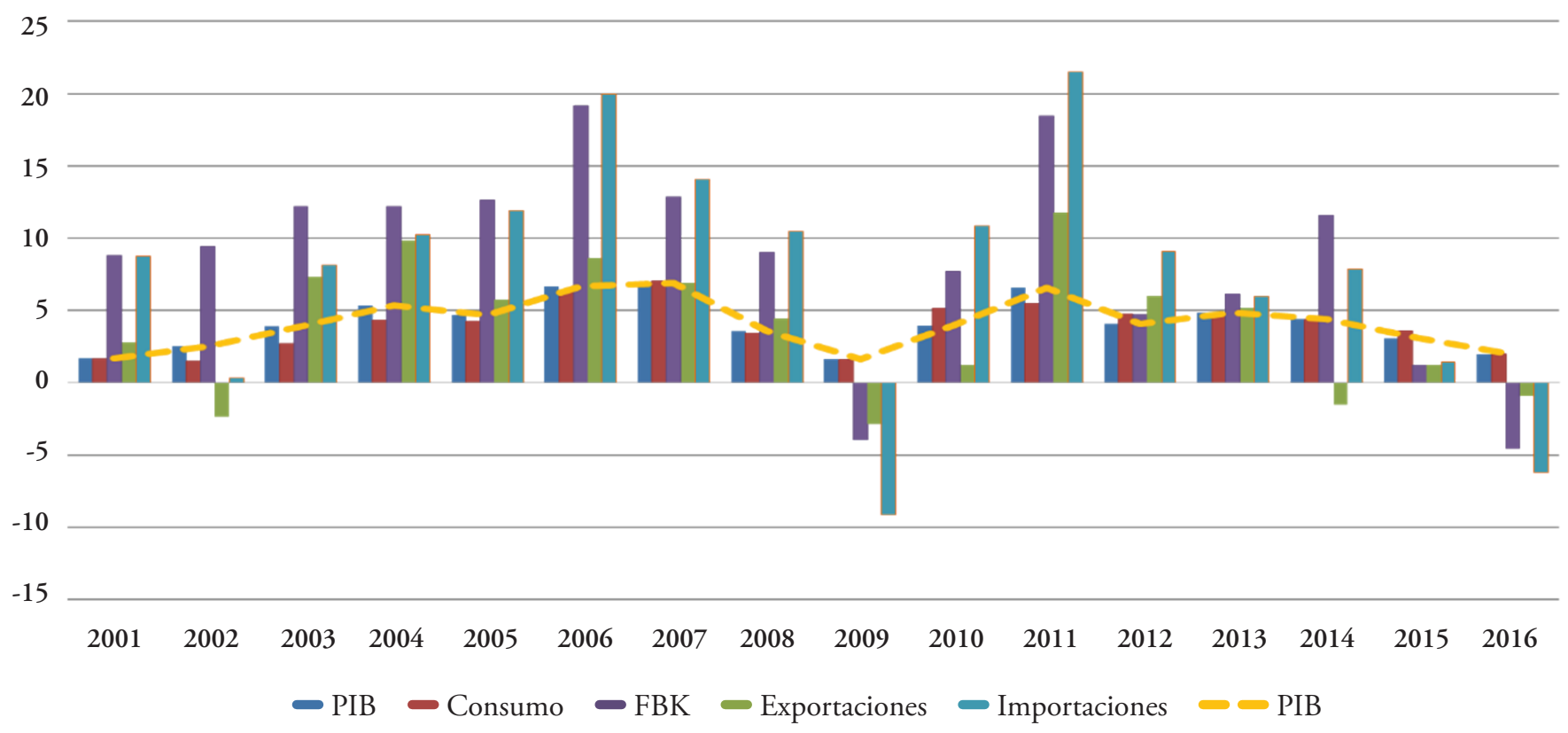

Fuente: Departamento Nacional de Estadística (DANE), 2018.

Si bien Colombia es un país con una vocación significativa para el sistema agrícola y la producción de materias primas, no ha logrado conseguir un modelo sostenible de "economía agrícola sostenible", sino más bien se ha fortalecido en los modelos extractivos relacionados con la minería, el petróleo, el gas o productos que no necesitan procesamiento para ahorrar en costos de producción. Así las cosas, los modelos de economía sostenible demandan, inicialmente, medios para investigación y aprovechamiento de los recursos naturales sin afectar ni alterar las especies que allí residen, para que a la vuelta de un periodo de tiempo prudencial se comiencen a percibir los 
beneficios económicos. El mejor caso, para hacer referencia de este modelo, se da en España, gracias a la Ley 2 de 2011 la cual entiende por economía sostenible:

un patrón de crecimiento que concilie el desarrollo económico, social y ambiental en una economía productiva y competitiva, que favorezca el empleo de calidad, la igualdad de oportunidades y la cohesión social, y que garantice el respeto ambiental y el uso racional de los recursos naturales, de forma que permita satisfacer las necesidades de las generaciones presentes sin comprometer las posibilidades de las generaciones futuras para atender sus propias necesidades (Jefatura del Estado, 2011, p. 10).

Si se analiza detalladamente, la visión de la Ley 2 logra unir las visiones del DS, permitiendo que avance positivamente por medio de las garantías que el Gobierno ha implementado, lo cual asegura la sostenibilidad económica del Estado, la sociedad y el ambiente. Finalmente, y en contraste con Colombia, se hace evidente la falta de visión central, que analice y consolide ampliamente los temas económicos, ambientales y sociales, para de esta forma arrojar el mejor modelo económico que más se asemeja y adapta a las necesidades del país.

\section{Análisis desde la visión social y educativa}

Uno de los puntos que más contiendas desencadena es la desigualdad entre la economía y la sociedad, dicha percepción no está del todo equivocada, tampoco es reciente; "desde los tempranos debates a fines de la década de 1970, se apuntó a que una desigual apropiación de los recursos naturales está asociada a la desigualdad social y económica" (Gudynas, 2011, p. 20). Estas discusiones han frenado sustancialmente el surgimiento de la economía solidaria y social, limitando el crecimiento en los sectores donde las clases medias y bajas se concentran mayoritariamente.
Como consecuencia, las relaciones de capital económico y calidad de vida se han estrechado a niveles insospechados, gracias a la inoperancia pública de las instituciones del Estado. Colombia, al igual que el resto de América Latina, ha sufrido este cambio, contrario a lo que realmente necesita la sociedad, en dónde los recursos económicos supeditan el nivel de vida, sin posibilidades de garantizar unos mínimos vitales al conjunto de la sociedad.

El deterioro acumulado sobre todo golpea a las clases media y en particular a los más pobres, por ejemplo, con alimentos de mala calidad, vivir en sitios contaminados, pérdida del patrimonio natural representado por los suelos o el agua, etc. Entretanto, la concentración de riqueza hace que las clases altas puedan "comprar" condiciones de vida de mayor calidad (Gudynas, 2003, p. 18).

En definitiva, acrecentando las condiciones de pobreza y desigualdad en sociedades que tradicionalmente han sido desiguales, pero en donde el Gobierno ha tratado de disminuir esta brecha.

El debate se cierne en relación con desniveles en lo social y lo económico, "en una sociedad crecientemente regida por el interés de lucro individual, no es fácil hacer compatible esa motivación con la conservación y buen uso de los recursos naturales" (Rodríguez Becerra, 1994, p. 15). Aquellos grupos sociales que cuenten con los recursos económicos pueden garantizar una calidad de vida excelente, en comparación con los menos favorecidos, que deben soportar la ineficiencia en las políticas públicas o estar rezagados en la cadena de formación, por ende, en sus condiciones de calidad de vida, replicando y perpetuando esta situación en las generaciones futuras.

Desde la orilla social se debe propender por el interés general, el cual tiene como ejes el bienestar sanitario, la construcción de un sistema de trabajo y pensión, brindar garantías para la calidad de vida, 
educación y formación en todas las etapas de la vida, entre otros. Contrapuesto a esta situación han surgido fenómenos que hacen difícil el manejo de las sociedades: la desigualdad de salarios, la limitación para las mujeres y su disparidad laboral. Uno de los mejores ejemplos de ello es el caso del desempleo a nivel nacional, que ha disminuido en los últimos años, pero vuelve a tener tendencias al alza, promoviendo el trabajo informal, con bajas condiciones de calidad o dando paso al desempleo.

\section{Gráfico 2. Tasa de desempleo en Colombia}

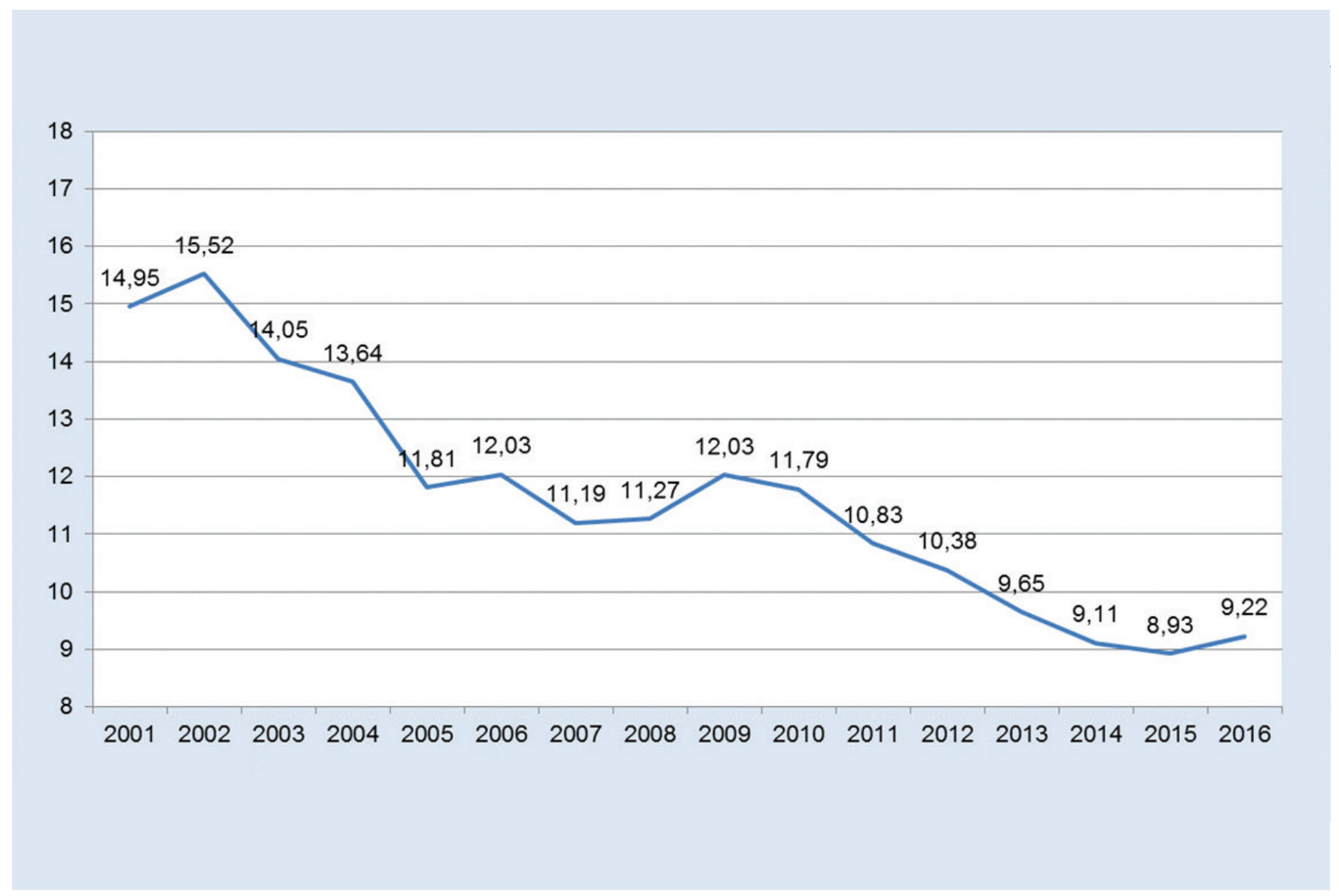

Fuente: DANE, 2016.

Como resultado se evidencia un serio desnivel entre economía y sociedad, donde la segunda se encuentra condicionada a la primera. Esto genera que los bienes y servicios puedan ser accesibles para un grupo limitado de personas; para los demás, deberán esforzarse mucho o esperar a que dichos productos o servicios reduzcan sus costos, con el riesgo de que la calidad se vea disminuida. Es decir, que la situación actual se presta para que las personas no puedan acceder a los mismos bienes.
Desde el campo de la educación la temática se concentra en los centros educativos y su potestad para adecuar los contenidos programáticos que en ellos se imparten y definir si optan o no por dictar materias relacionadas al DS. De acuerdo con el ejercicio de investigación adelantado, es posible evidenciar que, de las 66 universidades acreditadas, con calificación alta o en proceso de acreditación, existe paridad en relación con la calidad impartida en esta muestra, demostrando que existe competencia por 
llegar al público y por adelantar procesos de investigación. La especificación es la siguiente:

Tabla 1. Instituciones de educación superior acreditadas, oficiales y privadas

\begin{tabular}{lll}
\hline \multicolumn{1}{c}{ Sector } & \multicolumn{1}{c}{ Número } & \multicolumn{1}{c}{$\%$} \\
\hline PÚBLICO & 30 & $45 \%$ \\
PRIVADO & 36 & $55 \%$ \\
Total, Instituciones acreditadas & 66 & $100 \%$
\end{tabular}

Fuente: Cálculos propios con datos de (SNIES), Ministerio de Educación Nacional de Colombia, 2015.

Existe una regionalización de la educación en Colombia, forzando a las personas a que se desplacen a los centros urbanos para recibir los niveles de formación superiores, generando condiciones de desigualdad en la sociedad. A su vez, se afectan las condiciones de las universidades regionales a la hora de impartir modelos acordes a las necesidades de la región, como es el caso de Universidad Nacional - Sede Amazonas, la cual se ha enfocado en la sostenibilidad ambiental selvática; en comparación a la Universidad Nacional - Sede San Andrés y Providencia, que se concentra en la riqueza marina y la sostenibilidad del Caribe colombiano.

La gráfica 4 presenta la relación de los departamentos con la cantidad de universidades acreditadas vigentes. Demuestra que en solo 16 de los 32 departamentos de Colombia existen instituciones calificadas que implementan modelos de DS en su formación, al interior de diferentes programas. Esta realidad plantea que departamentos como Vaupés, Cesar, Chocó y Guainía, entre otros, no cuentan con acceso a una educación de calidad.

\section{Gráfica 3. Universidades acreditadas por región en Colombia}

\section{Instituciones de educación superior con acreditación de Alta Calidad, por departamento}

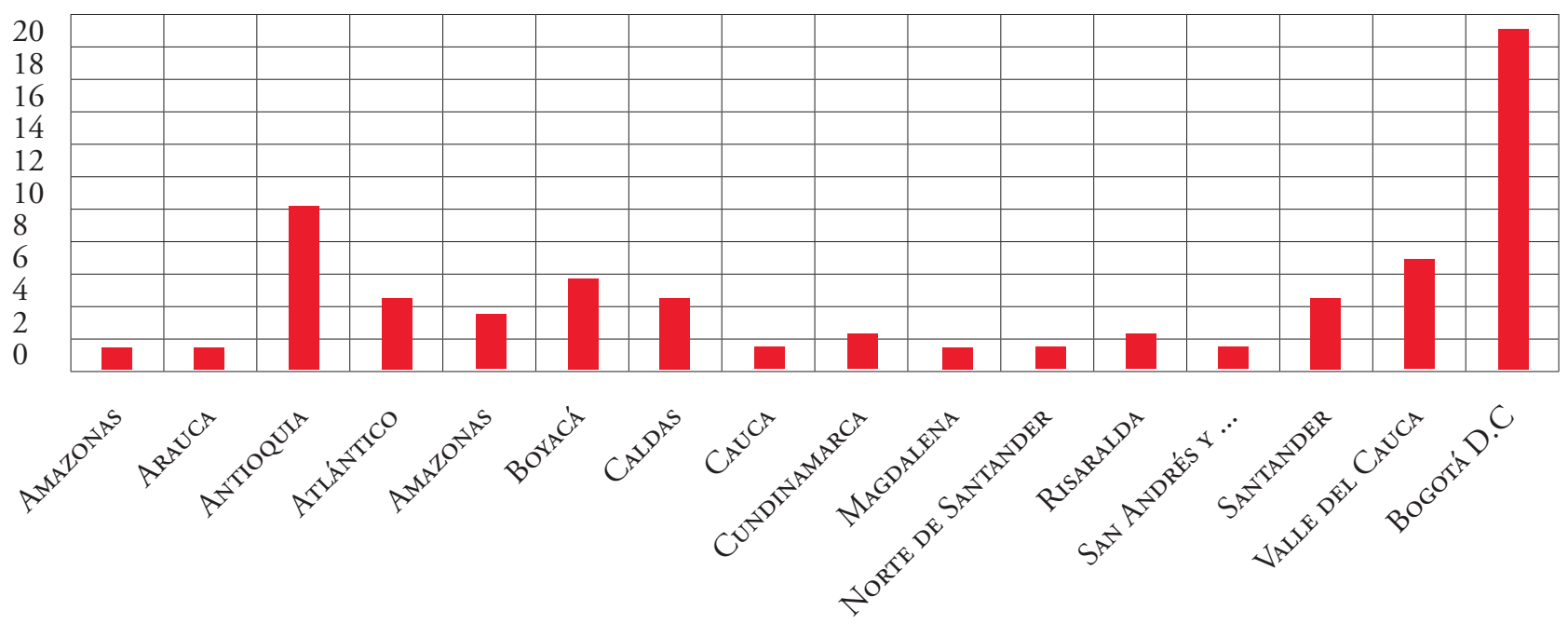

Fuente: Elaboración propia. 
Visiones y acercamiento del desarrollo sostenible en República Dominicana, complemento de la economía con educación

República Dominicana es un país ubicado en la zona central del mar Caribe, de acuerdo a la disposición de las Antillas, la capital es Santo Domingo, su estructura administrativa se divide en tres grandes regiones que albergan cerca de diez millones de habitantes ( «República Dominicana alcanzará los 10 millones de habitantes el 15 de septiembre», 2015), de acuerdo a estimaciones de la Oficina Nacional de Estadística (ONE). República Dominicana es un emblema de América, gracias a su riqueza cultural e histórica, que ha marcado el desarrollo político y social del Estado y su nación.
Como en la mayoría de los países latinoamericanos, la rama ejecutiva tiende a concentrar el poder y a dictar lineamientos para que los ministerios diseñen políticas en sus respectivos sectores. Sin embargo, en contraste con Colombia, República Dominicana tiene una economía estable y con un crecimiento acelerado, sin depender del petróleo y sus derivados. $\mathrm{Su}$ riqueza reside en el potencial turístico y en los recursos naturales. Además, recientemente las remesas, junto a algunos recursos provenientes del sector minero y del sector servicios, han demostrado tener un valor agregado para la economía local, impulsando el crecimiento del PIB sustancialmente, permitiéndole reducir la pobreza de manera sostenida, fundamentados en datos del Banco Central de República Dominicana («República Dominicana», 2018).

\section{Gráfica 4: Producto Interno Bruto - Per Cápita de República Dominicana}

\section{Evolución: PIB per capita de la República Dominicana}

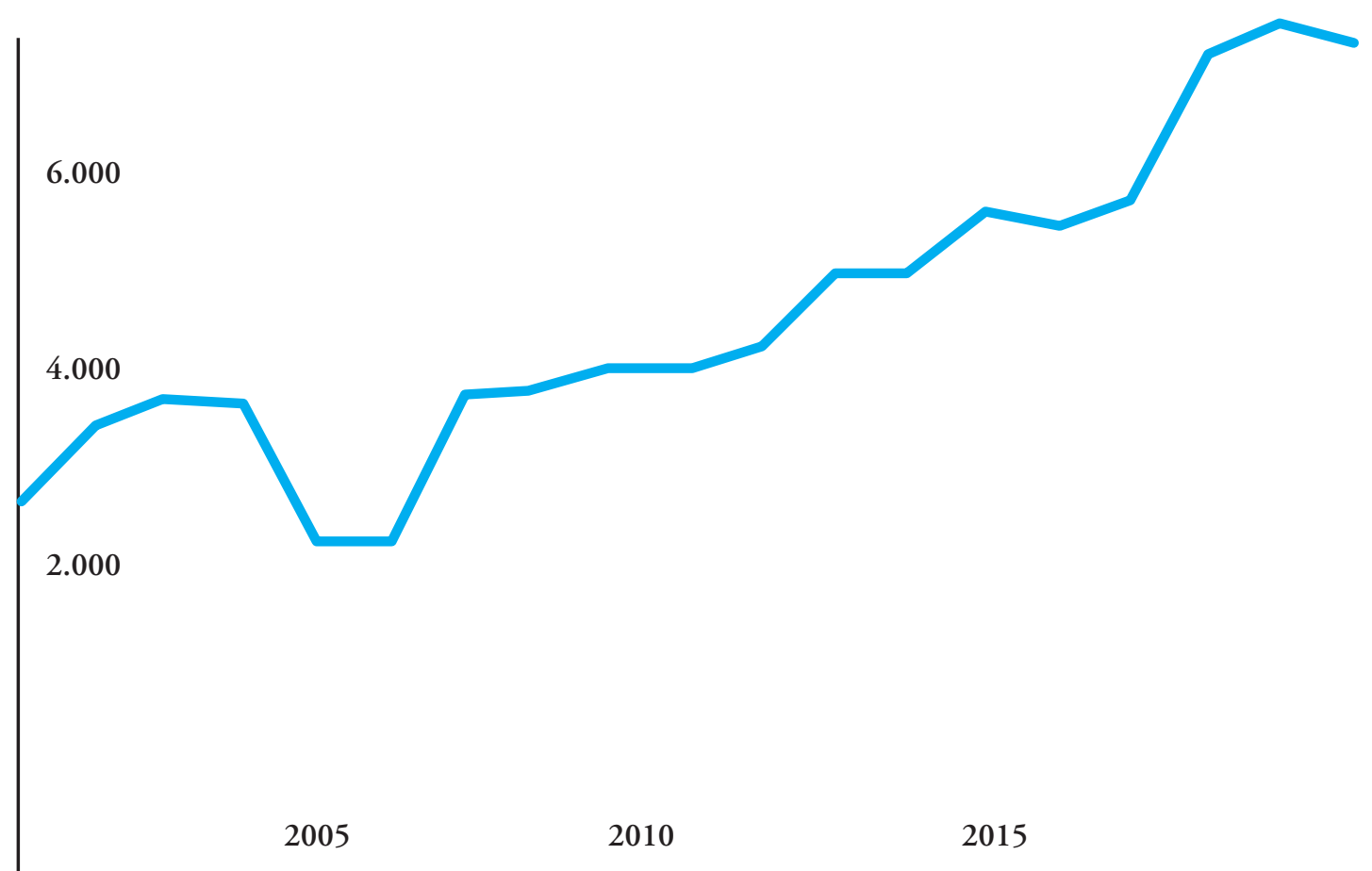

- PIB Per Capita

Fuente: Banco Mundial. 
Esta orientación económica, donde la agricultura es el segundo sector más importante, a lo largo de su historia, y que solo es superado por el turismo, ha permitido que el enfoque del DS en el país sea especialmente diseñado para el primer sector (Cassá, 1991). En consecuencia, el DS se ha enfocado hacia un turismo sostenible, en donde el principal músculo de la economía se ha focalizado para que se desarrollen planes ambientales sin afectar la productividad del país (Agüera, Guzmán \& Verdugo, 2015; Artigas, Batlle \& Del Olmo, 2005; Castellanos Verdugo \& Orgaz Agüera, 2013; Villareal \& Van Der Horst, 2008).

Uno de los estudios más importantes sobre la biodiversidad del país arroja conclusiones interesantes sobre la formación de los jóvenes alrededor de posturas sostenibles, vinculándose principalmente con la infraestructura sostenible y los servicios del área de conservación del medio ambiente (Morales, 1998). Además, corrobora que uno de los ejes fuertes con los que debe contar el país es:

Programa de educación ambiental: Su misión es la de educar a las comunidades locales y a los visitantes sobre la protección y conservación de los recursos naturales, la convivencia armónica hombre-naturaleza, lo mismo que contribuir a una concientización nacional ambiental. De una buena política de educación ambiental va a depender en gran medida el éxito de un país en su esfuerzo por la conservación de la naturaleza, fuente inagotable de recursos, cuando se maneja con criterio de sostenibilidad (p. 11).

La perspectiva legal evidencia los esfuerzos por parte de los diferentes Gobiernos en materia educativa, la Ley 66 de 1997 es la encargada de regir los modelos de educación bajo amparo de la Secretaría de Estado de Educación y el Ministerio de Educación. Por su parte, la Ley de Educación Superior de República Dominicana de 2001, crea el Sistema Nacional de Educación Superior, Ciencia y Tecnología, en ambos casos la ley sienta las bases del sistema de investigación científica nacional, los modelos de formación profesoral que funciona actualmente en el país (Congreso Nacional, 1997, p. 66).

Como consecuencia de esta postura, República Dominicana se ha comprometido fuertemente en aumentar la inversión en el sector educación en los últimos años, tratando de cumplir los objetivos de Dakar del año 2000 sobre la enseñanza, la educación y el aprendizaje, permitiéndoles ganar reconocimiento por parte de la UNESCO en el componente de educación sostenible, con apoyo a la industria turística y la protección del medio ambiente a lo largo de todo el territorio.

Sin embargo, las prioridades de los avances en el tema de la educación se concentran en desarrollar ejes de cooperación entre el Marco de Asistencia de Naciones Unidas para el Desarrollo (UNDAF), la UNESCO y la Estrategia Nacional de Desarrollo (END) con objetivo a 2030, buscando garantizar plenamente el derecho a la educación de calidad para todos, universalidad del aseguramiento en salud y vivienda digna, reconociéndolos como fundamentales y básicos en la sociedad. Además, el diseño de cátedras es esencial para responder a las necesidades locales y regionales, la cátedra INNOVEMOS o la cátedra UNESCO, son escenarios para la discusión de temas trasversales relacionados al DS (UNESCO, 2014, p. 27).

Otra perspectiva que se debe considerar es la que plantea internamente el Ministerio de Educación Superior, Ciencia y Tecnología desde el 2015, en relación con la normativa para la formación docente de calidad en la República Dominicana, en la que plantea a grandes rasgos una relación horizontal con el uso de herramientas virtuales junto a entornos sostenibles, para que los estudiantes desarrollen competencias mejores en todas las ramas del conocimiento (Ministerio de Educación Superior, Ciencia y Tecnología, 2015). Por medio de planes y proyectos el Gobierno pretende vincular diferentes entidades para que la apropiación del conocimiento aporte conscientemente a la sociedad dominicana. 
Lo que se puede analizar de la situación dominicana respecto a los ODS, es que se ha desarrollado una visión de país para el 2030, visualizándose como un país próspero en donde la ciudadanía viva dignamente, respetando el medio ambiente y con garantías económicas para su libre desarrollo. Es por ello qu e la visión se mat erializa en:

\section{Gráfica 5. Visión de país en República Dominicana}

\begin{tabular}{|c|c|c|c|c|c|}
\hline \multicolumn{6}{|c|}{ ¿Cómo conseguir la Visión País de largo plazo? } \\
\hline \multirow{6}{*}{$\begin{array}{l}\text { Políticas } \\
\text { Públicas }\end{array}$} & \multirow{6}{*}{$\begin{array}{l}\text { Fomentan y } \\
\text { refuerzan los } \\
\text { valores }\end{array}$} & Educación & Laboriosidad & \multirow{6}{*}{ Que propician } & Convivencia Pacífica \\
\hline & & Honestidad & Respeto & & Cohesión Social \\
\hline & & \multicolumn{2}{|c|}{ Solidaridad } & & \multirow{3}{*}{$\begin{array}{c}\text { Espíritu de } \\
\text { Superación personal }\end{array}$} \\
\hline & & \multirow{2}{*}{ Buen gobierno } & Justicia & & \\
\hline & & & Honradez & & \\
\hline & & \multicolumn{2}{|c|}{ Responsabilidad } & & Desarrollo colectivo \\
\hline
\end{tabular}

Fuente: (Montás, s. f.).

La consecución de visión de país se ha enfocado en la modernización del modelo educativo, para la correcta obtención de objetivos sociales sostenibles vinculantes con el medio ambiente y la economía. Sin embargo, las limitaciones del Estado para gestionar recursos, las prácticas corruptas, la ausencia de planes de ordenamiento territorial y ambiental afectan el cumplimiento de la agenda horizontal y multisectorial en el país, convirtiéndose en retos para el Gobierno.

\section{Danilo Medina, la visión 2012-2016 y 2016- 2020, el cambio continúa}

El papel central del actual presidente Danilo Medina para la priorización y el diseño de políticas públicas enfocadas en la educación, es uno de los pilares fundamentales planteados en su gobierno, para atender las necesidades sociales en distintos niveles. Sin embargo, el eje educativo no se presenta como un eje exclusivo, se compone por varios elementos igual de importantes que podrían limitar el impacto o el papel central de la educación. De acuerdo a la visión general del plan de gobierno
"Forjando Un Nuevo Modelo De Desarrollo Para La Prosperidad, La Inclusión Social Y Una Vida Mejor" (Medina, 2012, p. 5), demuestra que la visión del modelo de desarrollo está presente y latente de manera transversal en las propuestas del presidente.

La educación para el primer periodo presidencial 2012-2016, se presenta como un motor para fortalecer las capacidades y el ejercicio de la ciudadanía, haciéndola incluyente, solidaria y sin discriminación. Es por ello que las propuestas centrales en este eje se concentran en:

1. Destinar al menos el $4 \%$ del PIB al sector educativo, para concretar los objetivos.

2. La educación inicial, básica y media como motor para desarrollar el espíritu ciudadano solidario y activo.

3. Ampliar la cobertura y la prioridad de acceso para las comunidades pobres.

4. Eliminar el analfabetismo en los primeros años de gobierno. 
5. Fomentar la innovación, la investigación y la experimentación educativas.

En ese orden de ideas, el esfuerzo en materia educativa nos deja entre ver fácilmente la relación con el DS, gracias a que el eje de poder local, descentralización y participación del plan de gobierno se enfoca en el impacto territorial, el ordenamiento territorial y la relación con el entorno, situando la visión sobre el desarrollo sostenible nacional.

En contraste con el plan vigente para el segundo gobierno de Danilo Medina, la postura presidencial sobre el papel de la educación es horizontal y evidencia el papel de la comunidad internacional al interior de República Dominicana, en materia de diseño y ejecución de políticas públicas, estableciéndolo así:

Nuestro Programa es un compromiso para todo el gobierno y una convocatoria a toda la sociedad. Es una evidencia de que sí podemos seguir construyendo juntos ese mejor país que hemos sońado y cuyo horizonte está delineado en la Estrategia Nacional de Desarrollo para el año 2030, y en los Objetivos de Desarrollo Sostenible acordados en el marco del sistema de Naciones Unidas (Medina, 2016, p. 7).

Además, los planteamientos sobre el DS ahora se vinculan con ejes como la oferta turística, minería e hidrocarburos, racionalidad en la administración pública y la política exterior, todos como un área que demanda responsabilidad y profesionalización en las actuaciones del Estado a la hora de mitigar los posibles dańos o mejorar las condiciones actuales. Así pues, el sistema educativo sigue siendo en eje central para la salida de la pobreza y aumento de la clase media, continuando con los aportes significativos del PIB a este sector.

Es importante resaltar que la educación preuniversitaria desempeña un rol significativo, en la medida en que se preocupa por priorizar la atención en salud y en brindar provisiones de alimentación a las comunidades que se vean beneficiadas. Con el aumento de la cobertura educativa, es decir, un aumento de los estudiantes inscritos en centros educativos, se pretende mejorar el sistema de educación pública con profesores de calidad, ofreciendo programas de habilidades para la vida, educación sexual, acompañamiento psicopedagógico y creación de oportunidades económicas en la población joven, vinculando iniciativas medioambientales, económicas sostenibles y multidisciplinares.

Además, se ha logrado adelantar el proceso de reforma educativa gracias a una postura política, que evidencia voluntad por parte del ejecutivo en la priorización del tema, con vertientes del DS, vinculando más estudiantes, más maestros, más plazas disponibles y más materias con orientación sostenible, dentro del marco de una jornada extendida de calidad, con enfoque técnico y posibilidades de becas nacionales o extranjeras a lo largo del territorio nacional.

\section{Conclusiones}

El DS es un tema fundamental en la estructura social, económica y medioambiental, que se vincula con la educación de manera transversal. Tanto para la sociedad dominicana como para la colombiana el debate está en proceso de construcción, respondiendo a necesidades peculiares propias de cada caso. Si bien ya se superó la discusión sobre la adopción de los postulados por medio de la vinculación a políticas públicas locales y planes de gobierno, aún se encuentra en las fases iniciales de aplicación y adaptación, por ende, los resultados son mucho más demorados y puede que las acciones no se logren materializar a lo largo de su implementación, tanto por factores internos como externos.

Sin lugar a dudas, el tema educativo resulta subvalorado en algunos escenarios. Puntualmente, en el caso dominicano, la educación con enfoque sostenible y pionera en el desarrollo de proyectos vinculantes es solo una vaga idea del gobierno actual, que solo hasta 2016 lo plantea como necesario. Por su parte, 
el caso de Colombia no escapa a esta realidad; un Estado que no es capaz de defender modelos educativos sostenibles y que, en la actualidad, no garantiza educación de calidad en las regiones. Es un Estado que perpetúa la desigualdad y motiva las movilizaciones sociales como medio para superar la pobreza.

Es por ello que el nuevo debate se concentra sobre los tres ejes ya mencionados anteriormente. En los dos casos de estudio se parte del interés económico, para que desde allí se pueda garantizar la sostenibilidad educativa, asegurando derechos y desarrollando incentivos que permitan convertir a la educación en el eje central de la estructura social, con el desarrollo consciente del ejercicio de ciudadanía y que rompa brechas entre diferentes grupos sociales. En contraste, los casos en donde las posturas económicas han imperado y además hay ausencia o debilidad de la ley, se ha evidenciado que tanto las relaciones sociales como las medioambientales permanecen sin cambios significativos desde la calidad, conservando los riesgos de violación y vulneración de derechos, afectando de forma colateral la calidad y la cobertura de la educación impartida. Es decir, que para que se pueda reflejar algún cambio favorable se debe partir por un modelo de igualdad entre las características del DS para que trabajen mutuamente y arrojen resultados en conjunto.

Además, se encuentra un símil en los casos abordados en la medida en que las figuras diferentes al poder central, cuentan con un rol significativo a la hora de incentivar los OBS, asesorando y apoyando al presidente a la hora de diseñar políticas inclusivas y transversales que implementen $y$ atiendan integralmente a las necesidades sociales. Si bien, varían los sectores en donde se presentan estos fenómenos, son considerablemente cercanos a la economía central de los países. La situación cambia en la medida en que el caso dominicano evidencia una sinergia mayor y mejor estructurada con el medio ambiente, frente al caso colombiano y el sector extractivo.
La agenda educativa de República Dominicana logra convocar entidades internacionales para construir espacios de debate y aprendizaje con relación al entorno y a las necesidades sociales, demostrando que es un tema trascendental y crucial para el panorama político del país. En contraste, Colombia atraviesa una crisis de acceso a la educación en el marco de unas claras condiciones de desigualdad regional a lo largo del territorio, afectando el desarrollo nacional sobre el DS en las aulas.

En ese orden de ideas, resulta interesante analizar con detenimiento el caso dominicano en la medida en que los aportes a los ODS se concentran en materia medioambiental, sin dejar de lado temas económicos y sociales en el marco de un modelo educativo de calidad. La visión legal se concentra en la reciente Constitución promulgada en el 2010, donde el Estado tiene las potestades para impulsar el modelo sostenible con apoyo de empresas e instituciones privadas.

Finalmente, resulta valioso plantear que el enfoque educativo de República Dominicana se orienta prioritariamente en la educación inicial, básica y media, mientras que en Colombia se desarrolla una política fuerte en la educación superior, sin dejar de lado los temas de cobertura y acceso. Además, las estructuras orgánicas del Estado se han sabido enfocar en la cadena formativa con énfasis en la calidad, por medio de planes integrales desarrollados desde los ministerios de educación.

La educación universitaria en República Dominicana cuenta con los recursos financieros y enfoques sociales para ser el enlace correcto entre la educación escolar básica y la profesionalización o tecnificación de los ciudadanos, evidenciando que el actual gobierno se preocupa por avanzar en la formación educativa con calidad y cobertura. En paralelo, Colombia tiene recursos limitados y variables para el sector, en donde la educación superior se ve como un elemento de difícil acceso y la tecnificación ha ocupado más espacio en la formación de mano de obra. 


\section{Referencias}

Agüera, F. O., Guzmán, J. A. A., \& Verdugo, M. C. (2015). Turismo, desarrollo sostenible y percepción de los stakeholders. Un estudio de caso en República Dominicana. Economía del Caribe, (15), 1.

Antequera, J., González, E., \& Ríos Osorio, L. (2005). Sostenibilidady desarrollo sostenible: un modelo por construir. Recuperado de https:// bit.ly/2qKO4pE

Artigas, R. C., Batlle, J. R. M., \& Del Olmo, F. D. (2005). Desarrollo sostenible y medio ambiente en República Dominicana: medios naturales, manejo histórico, conservación y protección. Madrid, España. Universidad de Sevilla.

Asamblea Nacional Revisora. (26 de enero de 2010). Por medio de la cual se adopta la Constitución de la República Dominicana y se dictan otras disposiciones. Recuperado de https://bit. ly/1hbGY6d

Brundtland, G. H. (1987). Informe Brundtland. Washington, Estados Unidos. Editorial OMS Washington.

Cassá, R. (1991). Historia social y económica de la República Dominicana (Vol. 2). Santo Domingo, D.N., República Dominicana. Editora Alfa y Omega.

Castellanos Verdugo, M., \& Orgaz Agüera, F. (2013). Potencialidades ecoturísticas de la República Dominicana. TURyDES, 6(14-23).

Comisión Económica para América Latina y el Caribe, N. (2012). Los países de renta media: un nuevo enfoque basado en brechas estructurales. Recuperado de: http://cort.as/-I5Zh

Congreso Nacional. (1997). Ley Orgánica de Educación de la República Dominicana [No. 66 97]. República Dominicana. Recuperado de: http:// cort.as/-I5Zr

DANE (2018). Producto Interno Bruto (PIB) Históricos. Recuperado de: http://cort. as/-Hmij

Gudynas, E. (2003). Ecología, economía y ética del desarrollo sostenible. Abya Yala. Recuperado de https://bit.ly/2qJRRUc
Gudynas, E. (2011). Desarrollo sostenible: una guía básica de conceptos y tendencias hacia otra economía. Otra Economía, 4(6), 43-66. Recuperado de: https://bit.ly/2zTeHgd

Jefatura del Estado. (4 de marzo de 2011). Ley 2 - 2011, de Economía Sostenible. Recuperado de https://bit.ly/2ML5hvO

Ministerio de Educación Nacional de Colombia. (2015). Sistema Nacional de Información de la Educación Superior. Recuperado de: http:// cort.as/-ED9Q

Medina, D. (2012). Plan de Gobierno 2012-2016, Pub. L. No. 2012, 53. Recuperado de https:// bit.ly/2OJJOAg

Medina, D. (2016). Plan de Gobierno 2016 - 2020, Pub. L. No. 2016, 53. Recuperado de https:// bit.ly/2OJJOAg

Ministerio deEducación Superior, Cienciay Tecnología. (9 de diciembre de 2015). Normativa para la Formación Docente de Calidad en la República Dominicana. Recuperado de: http://cort.as/-I5aF

Montás, J. (s. f). Desarrollo Sostenible en República Dominicana: retos y desafíos. Recuperado de https://bit.ly/2RXzWFf

Morales, B. T. (1998). La Biodiversidad y su Papel en el Desarrollo del Ecoturismo. Revista Geográfica, (124), 21-32.

República Dominicana alcanzará los 10 millones de habitantes el 15 de septiembre. (2015, septiembre 7). Recuperado de https://bit.ly/2QILIIR

República Dominicana: panorama general. (2018, septiembre 20). Recuperado de https://bit. ly/2TdqSNV

Rodríguez Becerra, M. (1994). El desarrollo sostenible: ¿utopia o realidad para Colombia? Recuperado de https://bit.ly/2S0ndBN

Treviño, A., \& Sánchez, J. M. (s. f). Enfoques de desarrollo sostenible y urbanismo. Recuperado de https://bit.ly/2OKwJqi

UNESCO. (2014). Documento de Programación de la UNESCO para República Dominicana (20132017). Recuperado de https://bit.ly/2K52iL0

Villareal, R., \& Van Der Horst, A. (2008). Estrategia de competitividad turística de la República Dominicana. ARA, Revista de Investigación en Turismo, 1(1), 15-28. 
Discusión de la aplicabilidad del desarrollo sostenible desde las políticas públicas en la República Dominicana y en Colombia según la adaptación educativa de la Agenda 2030

\section{Datos de filiación}

Jacqueline Murillo Garnica, Doctora en Literatura española e hispanoamericana de la Universidad de Salamanca, profesora contratada programa PAC del ISFODOSU - Instituto Superior de Formación Docente Salomé Ureña, Ureńa, República Dominicana. Con intereses investigativos en educación, estudios literarios y estudios afrocaribeños. ORCID: 0000-0003-31625131 